\title{
LITERATUR
}

\section{Persönlichkeiten und Netzwerke deutscher Europapolitik}

\author{
Jürgen Elvert*
}

In beiden hier vorzustellenden Werken geht es um Akteure und deren Rolle in Organisationen, die sich nach dem Zweiten Weltkrieg der europäischen Einigung verschrieben hatten. Vanessa Conze untersucht mit der Abendländischen Bewegung und der EuropaUnion zwei Vereinigungen, die als Institutionen zwar klar umrissene Europa-Programme besaßen, deren Mitglieder daran jedoch ganz unterschiedliche Erwartungen knüpften. Diese werden am Beispiel einiger ausgewählter Akteure herausgearbeitet. Dagegen steht in dem von Jürgen Mittag und Wolfgang Wessels herausgegebenem Band mit Friedrich Carl von Oppenheim von vornherein nur ein Repräsentant einer Organisation im Mittelpunkt, der zwischen 1958 und 1973 das Amt des Präsidenten der Europa-Union ausübte. In beiden Fällen liegt somit der Untersuchungsschwerpunkt in der Nachkriegszeit und reicht bis in die 1970er Jahre, also in jenen Zeitraum, in dem die Gründungsphase des europäischen Integrationsprozesses abgeschlossen war und die Konsolidierungsphase begann, die mit der Formulierung der Einheitlichen Europäischen Akte und der Verabschiedung des EU-Vertrages endete. Beide Bücher berücksichtigen jedoch auch die Zwischenkriegszeit als Präge- und Sozialisationsphase ihrer Protagonisten gebührend. Denn nur so konnten die Wurzeln der nachkriegszeitlichen deutschen Europarezeption freigelegt werden, um die Kontinuitäten, aber auch die Brüche im deutschen europäischen Denken des 20. Jahrhunderts herauszuarbeiten.

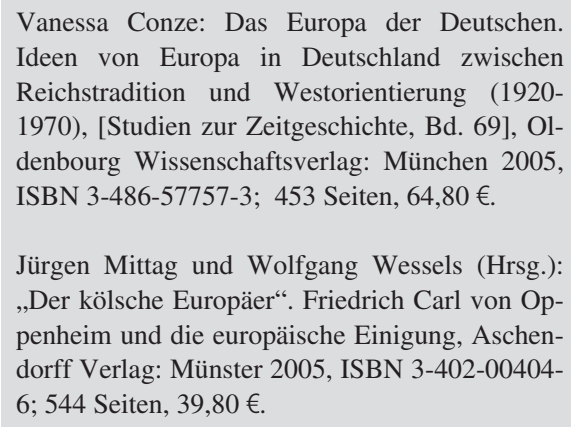

Jürgen Mittag und Wolfgang Wessels (Hrsg.): „Der kölsche Europäer“. Friedrich Carl von Oppenheim und die europäische Einigung, Aschendorff Verlag: Münster 2005, ISBN 3-402-004046; 544 Seiten, $39,80 €$.

\section{Die ,Abendländer}

Wenn Brüche insbesondere in der Arbeit von Vanessa Conze eine wichtige Rolle spielen sie spricht in diesem Zusammenhang mehrfach von „Wandel“ -, so hängt das natürlich mit der größeren programmatischen Breite zusammen, die die von ihr untersuchten Organisationen und deren Protagonisten vertraten. Berücksichtigt man die Affinitäten der ,Abendländer' zum Reichsdenken, wie es im Umfeld der Weimarer Konservativen Revolution gehegt wurde, reichten diese vom rechten antidemokratischen und antirepublikanischen Rand bis weit in das linksliberale republikanische Spektrum hinein, dem beispielsweise der „Verband für Europäische Verständigung“ zuzurechnen war. Freilich handelte es sich bei den Vertretern eines dezidiert auf eine Verständigung mit den westeuropäischen Siegermächten ausgerichteten deutschen Europadenkens der 1920er Jahre nur um eine kleine Minderheit, deren Stimme im Getöse nationalistischer und revisionistischer Agitation so

* Prof. Dr. Jürgen Elvert ist Professor für Geschichte des 19. und 20. Jahrhunderts (Schwerpunkt: Geschichte der Europäischen Integration) und für Didaktik der Geschichte an der Universität Köln. 
gut wie nicht gehört wurde. Stattdessen pflegten sie, wie Conze am Beispiel der Paneuropa-Union Coudenhove-Kalergis einerseits und des durch Wilhelm Heile vertretenen Verbandes für Europäische Verständigung andererseits zeigen kann, eine gegenseitige Abneigung und schwächten damit noch zusätzlich die ohnehin schwachen, auf gesamteuropäischen Ausgleich ohne revisionistische oder gar hegemoniale Hintergedanken zielenden Kräfte im Deutschen Reich.

Ob sich am Beispiel von zwei Organisationen das Europa der Deutschen insbesondere in der Nach-, aber auch in der Zwischenkriegszeit zeigen lässt, sei einmal dahingestellt. Bei der Wahl eines Buchtitels spielen eben immer auch Marketing-Gesichtspunkte eine Rolle. Unbestritten sei, dass Conze mit ihrer Untersuchung ein breites Spektrum deutschen europäischen Denkens im 20. Jahrhundert abdeckt. Die abendländische und die westeuropäische Bewegung stellen, so gesehen, die jeweiligen Mittelpunkte zweier sich nur wenig überschneidender Kreise dar, deren Radien, markiert durch die einschlägigen Vorstellungen ausgewählter, weil repräsentativer Protagonisten, bestimmte, in der deutschen Gesellschaft des Untersuchungszeitraums verbreitete Sichtweisen von Europa markieren.

Dass hier erhebliche Unterschiede im Denken der Zwischenkriegszeit einerseits und der Nachkriegszeit andererseits festzustellen sind, liegt vor dem Hintergrund der deutschen und europäischen Geschichte des 20. Jahrhunderts auf der Hand. Die deutsche Europarezeption der Zwischenkriegszeit war, wie auch Conze zeigt, in erheblichem Maße von einem Sonderbewusstsein geprägt, das dem Reich eine Führungsrolle in Mitteleuropa zudachte, und damit von einem diffusen Denkmodell, das sich im Verlauf des 19. Jahrhunderts im Dia$\log$ mit der Suche nach einer deutschen nationalen Identität entwickelt hatte. Die deutsche Kriegszieldiskussion vor dem und während des Ersten Weltkriegs hatte sich aus diesem Denken genauso gespeist wie die in diesen Jahren stattfindende Suche nach einer europä- ischen Friedensordnung, an der sich unter anderen Friedrich Naumann an prominenter Stelle beteiligte, dessen Mitteleuropa-Buch nach seinem Erscheinen 1915 für großes Aufsehen sorgte. War Naumann noch von einem föderalen und dem Prinzip der Gleichberechtigung gehorchenden Ordnungsmodell ausgegangen, neigte die einschlägige Diskussion nach 1918 eher dazu, Mitteleuropa als einen Raum zu betrachten, in dem das Deutsche Reich aufgrund seiner historischen, kulturellen und ökonomischen Bedeutung eine Führungsrolle zu spielen habe. In den letzten Jahren hat die Forschung nachgewiesen, dass diese Meinung sich als Mehrheitsmeinung im öffentlichen Bewusstsein seit Mitte der 1920er Jahre durchsetzen konnte und die nationalsozialistische Großraum-Ideologie hier nach 1933 problemlos anknüpfen konnte.

\section{Scharnierfunktion des Rheinlands}

Vanessa Conze bestätigt diesen Befund in ihrer Einleitung, zeigt aber auch, dass sich das Verhältnis der von ihr untersuchten Organisationen und Akteuren zum mitteleuropäischen Ordnungsmodell der 1920er Jahre unterschiedlich entwickelte. So stand Wilhelm Heile, einer der prominentesten Westeuropäer der Conze'schen Auswahl, als enger Mitarbeiter Friedrich Naumanns während des Ersten Weltkriegs erkennbar unter dem Eindruck von dessen mitteleuropäischen Konzepten. Zwar hatte er sich seit Anfang der 1920er Jahre zunehmend einer gesamteuropäischen Verständigung verschrieben, mit der er dem Deutschen Reich die völlige politische Gleichberechtigung sichern wollte. Langfristig sollte dies freilich die Voraussetzung für eine Realisierung der großdeutschen und mitteleuropäischen Ambitionen des Reiches sein - womit sich Heiles auf den ersten Blick so atypischer westeuropäischer Verständigungsansatz bei genauerem Hinsehen als eine weitere, wenngleich verschleierte Variante mitteleuropäischen Denkens entpuppt.

Dagegen ging es den Anhängern der Abendland-Idee jedenfalls in der ersten Hälfte der 
1920er Jahre um einen deutsch-französischen Ausgleich als Voraussetzung für die Verhinderung künftiger gegenseitiger Selbstzerfleischung auf dem Schlachtfeld. Viele von ihnen maßen dem Rheinland eine Art Scharnierfunktion zwischen beiden Nationen bei, da sie die Landschaften entlang des Stroms als Kernlande des Abendlandes verstanden, in dem sich das abendländische Reich karolingischer Provenienz verfestigt hätte. Ähnliches galt für das deutsch-polnische Verhältnis, für das der Katholizismus als das entscheidende Bindeglied angesehen wurde. Damit unterschieden sich die Abendländer erheblich vom politischen Mainstream der Zeit. Allerdings nahm die relative Offenheit gegenüber den europäischen Nachbarn im Westen wie im Osten seit etwa $1925 \mathrm{ab}$, seither dominierten auch in den abendländischen Ordnungsmodellen die mitteleuropäischen Elemente.

\section{Erfordernisse der Nachkriegszeit}

Das an die mitteleuropäischen Ordnungsmodelle anknüpfende Großraumdenken in nationalsozialistischer Zeit, der Zusammenbruch des NS-Staates sowie der systemische Konflikt bereiteten dem spezifisch deutschen Mitteleuropadenken ein Ende. Die bald nach Kriegsende einsetzenden Bemühungen um Ingangsetzung eines europäischen Integrationsprozesses schufen freilich neuen Raum für europäische Ordnungsmodelle. In diesem Zusammenhang konnten sowohl die Abendland-Bewegung als auch jene Kräfte rasch Gestaltungsspielraum erlangen, die sich in den 1920er Jahren aus anderen als aus abendländischen Motiven heraus um einen deutschwesteuropäischen Ausgleich bemüht hatten. Dass die Abendländer wieder versuchten, an die eigenen Überlegungen der Zeit kurz nach Ende des Ersten Weltkriegs anzuknüpfen, kann nicht überraschen, ebenso wenig die positive Resonanz darauf. Schließlich bot der Re-Christianisierungsgedanke Halt in einer Zeit, in der es neue moralische Maßstäbe zu bestimmen galt. Die Gründe für den erneuten Rechtsruck der Abendland-Bewegung unter dem Eindruck des sich verschärfenden Kalten
Krieges liegen Conze zufolge im Bemühen, neben moralischem auch an politischem Gewicht zu gewinnen. Doch hatten sich die Zeiten im Vergleich zu den 1920er Jahren verändert. Weder die Einrichtung der Abendländischen Akademie noch die Schaffung von Institutionen mit politikberatender und -beeinflussender Zielsetzung wie das Europäische Informations- und Dokumentationszentrum (CEDI) konnten einen kontinuierlichen Bedeutungsverlust dieser Bewegung verhindern. Und auch wenn Teile von ihr Anfang der 1970er Jahre in der Paneuropa-Union aufgingen, gelang es der Abendland-Bewegung nicht, sich dem pragmatischen Ansatz einer den konkreten Integrationsprozess stützenden Bewegung wie der Europa-Union als echte Alternative zu präsentieren.

\section{Ringen um die Monopolstellung im bundes-} deutschen Europadiskurs

An diesem Beispiel wird die Unvereinbarkeit jenes im 19. Jahrhundert entstandenen spezifisch deutschen Europadenkens mit den Erfordernissen der Nachkriegszeit besonders deutlich. Eben weil das NS-System sich so problemlos der Europakonzepte der 1920er Jahre bedienen konnte, waren diese nach 1945 diskreditiert und neue Ansätze gefordert. 1945 steht somit nicht nur für einen Wandel im Europadenken der Deutschen, sondern stellt auch in dieser Hinsicht eine veritable Bruchstelle dar. Ohne die Erfahrung von 1945 hätten sich die genuin westeuropäischen Denkansätze, die beispielsweise der Kölner Bankier Friedrich Carl von Oppenheim verkörperte, vermutlich nicht so rasch und nachhaltig durchsetzen können. Dass es sich dabei freilich nicht um einen reibungslosen Neuanfang handelte, sondern dieser vielmehr mit zahlreichen Problemen behaftet war, kann Vanessa Conze en detail und quellengestützt zeigen. Besonders interessant erscheint hier die Rivalität zwischen Europa-Union und Paneuropa-Union der 1950er und 1960er Jahre, in der mit harten Bandagen um die ,Monopolstellung ' im bundesdeutschen Europadiskurs gekämpft wurde. 
Dabei hatte sich, wie Wilfried Loth in seinem Beitrag über die Bedeutung der Europa-Union für den europäischen Integrationsprozess im Sammelband von Mittag und Wessels zeigt, diese anfangs zumindest indirekt auf die Hilfe der Paneuropa-Union stützen können, war sie doch in ihrer Gründungsphase unter anderem vom American Committee for United Europe finanziert worden und damit von einer Einrichtung, die auf eine Initiative CoudenhoveKalergis zurückging, dann allerdings rasch unter den Einfluss der CIA geriet. Auch wenn es unter der Präsidentschaft Eugen Kogons bis 1953 durchaus angestrebt wurde, entwickelte sich die Europa-Union niemals zu einer Massenbewegung, gleichwohl verfügte sie im bundesdeutschen politischen Raum der 1950er und 1960er Jahre über beträchtlichen Einfluss als Schnittstelle zwischen Politik, Wirtschaft und vornehmlich bürgerlicher Öffentlichkeit liberal-konservativer Prägung. Finanzielle Probleme der Anfangsjahre konnten, nachdem von Oppenheim 1954 das Amt des Schatzmeisters übernommen hatte, überwunden werden, seither konzentrierte sich die Europa-Unionsführung darauf, den Westintegrationskurs Adenauers zu stützen und die Öffentlichkeit durch gezielte Bildungsarbeit über Integrationsspezifika zu informieren. In den 1960er Jahren profilierte sie sich als entschiedene Gegnerin der de Gaulle'schen Europapolitik, nach dessen Rücktritt verblasste allerdings das öffentliche Interesse an der Europa-Union zusehends.

Oppenheims Wirken für die europäische Integration

Dass das jedoch nicht deren Ende bedeutete, unterstreicht Heinrich Schneider in seinem autobiograpfisch gehaltenen Beitrag. Der Autor erinnert sich an seine persönlichen Begegnungen mit Friedrich Carl von Oppenheim, die sich aus der gemeinsamen Tätigkeit für die Europa-Union ergaben. Dabei gelingt es ihm, deren Geschichte gleichsam mit Leben zu erfüllen. Denn auch wenn von Oppenheim im Zentrum der Betrachtung steht, begegnet man zahlreichen anderen Persönlichkeiten des öffentlichen und politischen Lebens der ersten drei Jahrzehnte bundesrepublikanischer Existenz und lernt so manches Neue über deren jeweilige Positionen zur europäischen Integration im Allgemeinen und Europa-Union im Speziellen kennen. Sie alle stehen im Schneider'schen Beitrag in einer Form des Dialogs mit von Oppenheim, dessen eigene facettenreiche, stets aber gewinnende Persönlichkeit im Laufe der Zeit dem Leser immer vertrauter wird.

Schneider stellt seinen Protagonisten somit anhand einzelner ausgewählter Episoden vor, Jürgen Mittag porträtiert auf gut 150 Seiten von Oppenheims Wirken für die europäische Integration vor seinem familiären und, damit eng verbunden, beruflichen Hintergrund. Väterlicherseits war dieser zwar durchaus national-konservativ geprägt, doch die US-amerikanische Herkunft seiner Mutter ebenso wie seine Berufsausbildung im Ausland verhinderte eine allzu enge Einbettung in das Meinungsklima der Weimarer Republik. Auch wenn er selbst dem nationalkonservativen Lager nahe stand, zählte er von vornherein zu den Kritikern des Nationalsozialismus. Die Nähe zu Widerstandskreisen führte schließlich im September 1944 zu seiner Verhaftung, ohne dass es noch zu einer Verhandlung gegen ihn gekommen wäre. Seinen eigenen Erinnerungen zufolge waren es die Erfahrungen während der Haft, welche ihn zu einem überzeugten Anhänger der europäischen Integration machten. Damit war sein Aufgabengebiet für die Nachkriegsjahre abgesteckt: Wiederaufbau seines Bankhauses, Konsolidierung des westdeutschen Wirtschaftssystems und tatkräftige Unterstützung der europäischen Integration durch Mitwirkung in der EuropaUnion, zunächst auf lokaler, später auf nationaler und europäischer Ebene - als ,kölscher Europäer".

Sein Wirken für die Kölner Europa-Union zeichnen Jost Dülffer und Jutta Cohausz detailliert und quellengestützt nach. Hier wird deutlich, dass von Oppenheim sein organisatorisches Talent ebenso wie seine Fähigkeit 
zum Krisenmanagement bereits erfolgreich ,vor Ort' erprobt hatte, bevor er es ab 1954 auf nationaler Verbandsebene einbringen musste. Für Wolfgang Wessels war das ein zentraler Bestandteil der Oppenheim'schen Strategie. Er war als „Akteur und Spieler“ Teil eines im ,europäischen Mehrebenensystem ' fest verankerten dichtmaschigen Netzwerkes, das Finanzwelt, Wirtschaft und Politik eng miteinander verband - weil es allen nützte.

\section{Der biografische Blick}

Vanessa Conzes „Das Europa der Deutschen“ ebenso wie der von Mittag und Wessels herausgegebene Sammelband „Der kölsche Eu- ropäer" stellen jeweils auf eigene Weise wertvolle Beiträge für die Erforschung der ersten Phase des europäischen Integrationsprozesses dar, insbesondere hinsichtlich der Bedeutung der Zusammensetzung der pro-europäischen Lobby der 1950er Jahre sowie der Wirkungen der Lobby-Arbeit. Beide Studien zeigen darüber hinaus eindrucksvoll, wie wichtig der - seit den 1970er Jahren unter dem Eindruck der Vorherrschaft sozialwissenschaftlicher und strukturgeschichtlicher Methoden in den Geschichtswissenschaften lange Zeit als überholt betrachtete - biografische Zugang gerade auch im Rahmen der europäischen Integrationsforschung ist.

\title{
Neue Ideen für die Grenzen Europas
}

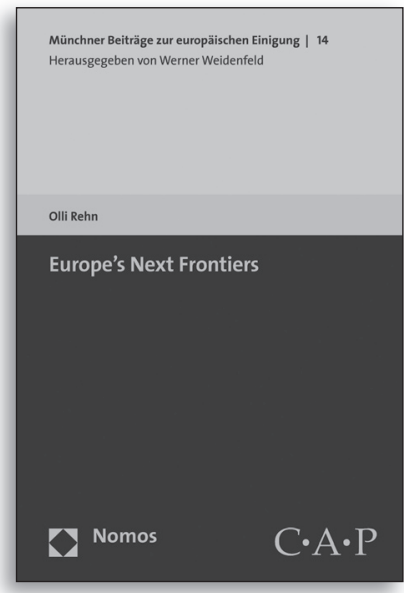

C) Nomos

\author{
Europe's Next Frontiers \\ Von Olli Rehn \\ 2006, 123 S., brosch., 17,90 €, ISBN 3-8329-2417-5 \\ Münchner Beiträge zur europäischen Einigung, Bd. 14 \\ Jahrhunderte lang waren wirtschaftliche und \\ geistige Offenheit entscheidend für die Entwick- \\ lung der Menschheit - eine Errungenschaft der \\ Aufklärung. Diese Offenheit muss Europa wieder \\ zurück gewinnen. \\ Olli Rehns Band ist ein Plädoyer für mehr Reform- \\ willen und der Aufruf, Veränderung in Europa als \\ etwas Positives zu begreifen.
}

Bitte bestellen Sie bei Ihrer Buchhandlung oder bei: Nomos Verlagsgesellschaft | 76520 Baden-Baden | www.nomos.de 